Check for updates

Cite this: RSC Adv., 2018, 8, 29338

\title{
Rational design of sequestered DNAzyme beacons to enable flexible control of catalytic activities $\dagger$
}

\author{
Wei Tang, (D) *a Mengxi Cheng, ${ }^{a}$ Danling Dai, ${ }^{a}$ Zhonghua Xiong ${ }^{\mathrm{a}}$ and Feng Liu (D) ${ }^{\mathrm{b}}$
}

DNAzymes as functional units play increasingly important roles for DNA nanotechnology, and fine control of the catalytic activities of DNAzymes is a crucial element in the design and construction of functional and dynamic devices. So far, attempts to control cleavage kinetics can be mainly achieved through varying the concentrations of the specific metal ions. Here we present a facile sequestered DNAzyme beacon strategy based on precisely blocking the catalytic core of the DNAzyme, which can flexibly regulate the DNAzyme cleavage kinetics without changing the concentrations of metal ions. This strategy can be extended to couple with a large number of other RNA-cleaving DNAzymes and was successfully applied in designing a dual stem-loop structure probe for arbitrary sequence biosensing, which provides the possibility of scaling up versatile and dynamic DNA devices that use DNAzymes as functional modules.

Received 6th July 2018

Accepted 8th August 2018

DOI: $10.1039 / c 8 r a 05757 e$

rsc.li/rsc-advances which limits the possibility of scaling up versatile and dynamic DNA devices that use DNAzymes as functional modules.

Herein, we propose a novel sequestered DNAzyme beacon strategy based on precisely blocking the catalytic cores of DNAzymes, which can flexibly control of the catalytic activities without changing the concentrations of metal ions. We explore the effects of the binding strength and the concentration of triggering stimulator on the cleavage kinetics. Furthermore, by applying the sequestered DNAzyme beacon strategy, we design a dual stem-loop structure probe to construct general biosensing systems for arbitrary target sequence. Taken together, these results demonstrate the possibility of flexibly designing more dynamic and controllable DNAzyme-based nanodevices.

ditional versatility over base-pair hybridization based nan devices and, because DNAzymes often catalyze multiple turnover reactions, such devices can have amplification effects. Thus, fine control of the catalytic activities of DNAzymes is a crucial element in the design and construction of functional and autonomous devices. Most DNAzymes consist of two binding arms and one catalytic core, activated via binding with unique metal ions such as $\mathrm{Pb}^{2+},{ }^{31,32} \mathrm{UO}_{2}{ }^{2+},{ }^{33} \mathrm{Zn}^{2+},{ }^{34,35} \mathrm{Na}^{+},{ }^{36}$ and $\mathrm{Cr}^{3+} \cdot{ }^{37}$ Attempts to control cleavage kinetics can be mainly achieved through varying the concentrations of the specific metal ions. However, it remains a major challenge to develop facile strategies for fine control of the catalytic activities of DNAzymes without changing the concentrations of metal ions,

\footnotetext{
${ }^{a}$ Institute of Materials, China Academy of Engineering Physics, Mianyang, 621700, China.E-mail: weitang@caep.cn

${ }^{b}$ Beijing National Laboratory for Molecular Sciences, Key Laboratory of Bioorganic Chemistry and Molecular Engineering of Ministry of Education, College of Chemistry and Molecular Engineering, Peking University, Beijing 100871, China

$\dagger$ Electronic supplementary information (ESI) available: Supporting tables and figures. See DOI: 10.1039/c8ra05757e
}

\section{Materials and methods}

\section{Reagents and chemicals}

All HPLC-purified DNA oligonucleotides were purchased from Sangon Inc. (Shanghai, China). The DNA sequences and modifications are listed in Table S1. $\dagger$ The secondary structures of all oligonucleotides, including the thermodynamic parameters, were calculated using NUPACK software (http:// www.nupack.org/). DNA stock solutions were prepared by dissolving oligonucleotides in TE buffer $(50 \mathrm{mM}$ Tris(hydroxymethyl)methyl aminomethane (Tris), $1 \mathrm{mM}$ ethylene diamine tetraacetic acid (EDTA); pH 8.0) and stored in the dark at $4{ }^{\circ} \mathrm{C}$ before use. Zinc chloride $\left(\mathrm{ZnCl}_{2}\right)$ and all other chemicals employed were of analytical grade and used without further purification. All solutions were prepared with ultrapure water, which was obtained through a Millipore Milli-Q water purification system (Molsheim, France) and had an electrical resistance of $\geq 18.2 \mathrm{M} \Omega$. 


\section{Time-dependent fluorescence measurements and kinetic analysis}

Sequestered DNAzyme beacons (SDB) were annealed in $50 \mathrm{mM}$ HEPES (pH 7.0) and $100 \mathrm{mM} \mathrm{NaCl}$ buffer by heating at $95{ }^{\circ} \mathrm{C}$ for $5 \mathrm{~min}$ and then slowly cooling to room temperature over 2 hours. Fluorescence measurements were performed by using a QM-40 fluorescence spectrometry (PTI, Canada) at $25{ }^{\circ} \mathrm{C}$. Sample solutions were excited at $522 \mathrm{~nm}$ (slit $2 \mathrm{~nm}$ ), and the emission signal was recorded with wavelength of $539 \mathrm{~nm}$ (slit 2 $\mathrm{nm})$. For all time-dependent kinetic measurements, $20 \mu \mathrm{L}$ of $10 \mathrm{mM} \mathrm{Zn}^{2+}$ (for 10-23 DNAZyme based system, $1 \mathrm{mM} \mathrm{Pb}^{2+}$ was used), $2 \mu \mathrm{L}$ of $50 \mu \mathrm{M}$ DNAzyme substrate (S), and $2 \mu \mathrm{L}$ of $10 \mu \mathrm{M}$ SDB were added one after another to buffer solution with a total volume of $200 \mu \mathrm{L}$. After thoroughly mixing the components, the fluorescence change were monitored in 60 s by fluorescence spectrometry. In all graphs, time $t=0$ indicates the time of SDB being added to the solutions. The enzyme-catalyzed reactions were assumed to follow the rate-law of pseudo-first order kinetics. The observed rate constant $k_{\text {obs }}$ was obtained by curve fitting using OriginPro 8 based on the equation $y=a+b \mathrm{e}^{-k x}$, where $k$ is the observed rate constant.

\section{Nucleic acids analysis}

To detect target DNA, $2 \mu \mathrm{L}$ of $50 \mu \mathrm{M} \mathrm{S}, 2 \mu \mathrm{L}$ of $10 \mu \mathrm{M}$ Probe sequence and $5 \mu \mathrm{L}$ of specific concentration of target DNA were added one after another to buffer solution with a total volume of $180 \mu \mathrm{L}$. After thoroughly mixing, the components were incubated at room temperature for $20 \mathrm{~min}$. A $20 \mu \mathrm{L}$ of $10 \mathrm{mM} \mathrm{Zn}^{2+}$ was added to induce the DNAzyme catalysed cleavage reaction. After vortexing to mix the components thoroughly, the fluorescence change was monitored in $60 \mathrm{~s}$ by fluorescence spectrometry. In all graphs, time $t=0$ indicates the time of $\mathrm{Zn}^{2+}$ being added to the solutions.

\section{Results and discussion}

\section{The mechanism of the sequestered DNAzyme beacons}

The mechanism of the controlled catalytic activities by designing sequestered DNAzyme beacons (SDBs) is illustrated

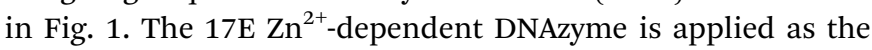

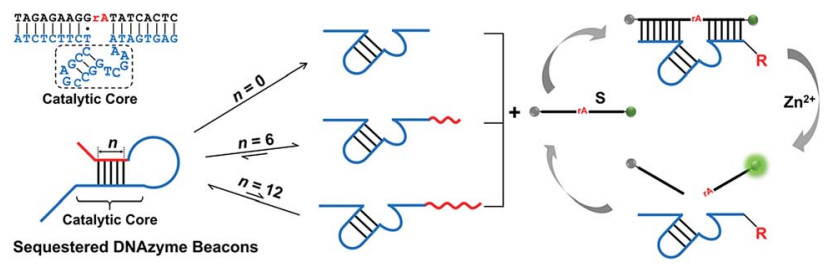

Fig. 1 Mechanism proposed for the sequestered DNAzyme beacons (SDBs) with controllable catalytic activities. Inset shows the secondary structure of $\mathrm{Zn}(॥)$-dependent deoxyribozyme-substrate complex 17E/ DS. SDB is constructed by 17E (blue domain) with an extended blocking sequence (red domain) attached at the $3^{\prime}$ end. The catalytic core of $17 \mathrm{E}$ is controllably sequestered in the stem of the hairpin structure. The hairpin structure of SDB can be reversibly unfolded. The $17 E$ in SDB can be then liberated and hybridize with a fluorophore and quencher labelled substrate to form a catalytic system. The nucleotide sequences of all strands are provided in Table S1 (ESI†). model biocatalyst in our systems. ${ }^{35}$ Similar to most of other in vitro selected DNAzymes, $17 \mathrm{E}$ is composed of a $14 \mathrm{nt}$ catalytic core and two binding arms. An extended blocking sequence with an overhung stick end (red domain) is introduced to the $3^{\prime}$ end of $17 \mathrm{E}$ (blue domain), which can hybridized partially with the catalytic core of $17 \mathrm{E}$. Thus, the catalytic core is controllably sequestered in the stem of the hairpin structure, resulting in the reducing of the cleavage reaction rate of $17 \mathrm{E}$. By modulating the binding strength of the stem, the cleavage rates can be tuned continuously. It is noteworthy that the sequestered DNAzyme beacon strategy presented here (1) is facile and do not need to change the component of the DNAzyme, (2) can be extended to couple with a large number of other RNA-cleaving DNAzymes, and (3) provides the possibility of scaling up DNA devices that use DNAzymes as functional modules, which could be applied to many field while metal ions cannot be readily changed, such as in vivo applications.

\section{Control of cleavage rate by modulating the binding strength} and hybridization state of the catalytic core

The relationship between the cleavage rate and the binding strength of the catalytic core was investigated. The $18 \mathrm{nt}$ substrate strand (S) was synthesized with a FAM fluorophore and a BHQ1 quencher at either end, serving as the signal reporter. The progress of the cleavage reaction can therefore be observed by monitoring the donor fluorescence intensity. In this work, the number of the hybridized bases in catalytic core is annotated by $n$. As shown in Fig. 2A, the reaction rate $\left(k_{\mathrm{obs}}\right)$ is the highest when $17 \mathrm{E}$ is free $\left(n=0, k_{\mathrm{obs}}=0.18 \mathrm{~min}^{-1}\right)$, and that is reduced by over one order of magnitude when 12 bases of the catalytic core are hybridized $\left(n=12, k_{\mathrm{obs}}=0.013 \mathrm{~min}^{-1}\right)$. The results reveals that by modulating the binding length $n$, the cleavage rates can be changed by over one order of magnitude.
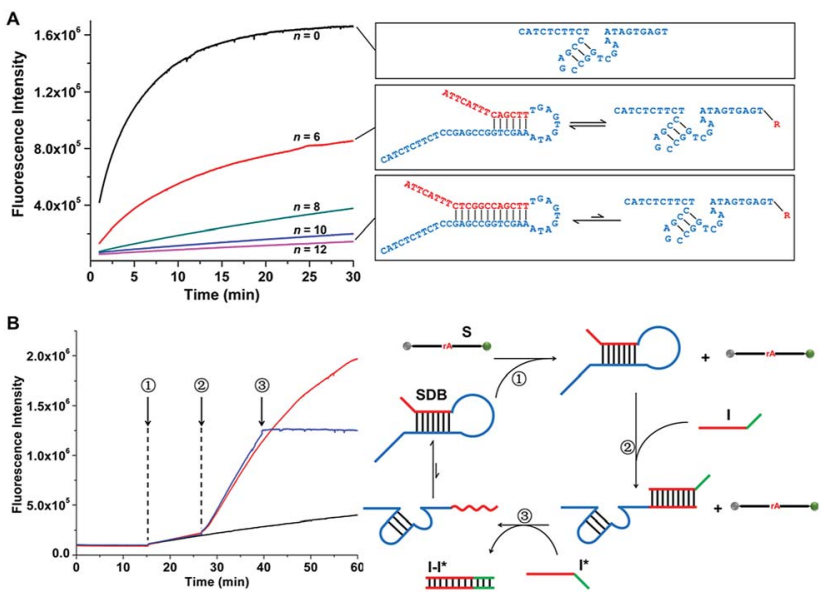

Fig. 2 Control of cleavage rates through control of binding strength of the catalytic core. (A) Catalytic cleavage reactions of SDBs with various binding length $n$. Initial concentrations: $1 \mathrm{mM} \mathrm{Zn}^{2+}, 100 \mathrm{nM} \mathrm{SDB}$, and $500 \mathrm{nM} \mathrm{S}$. (B) Dynamic control of cleavage reaction. All traces start from the addition of inactive SDBs $(n=12)$ into $S$ solutions. For the middle trace, a complementary initiator (I) was added. For the top trace, after the addition of $\mathrm{I}$, an anti-I sequence I* was added to

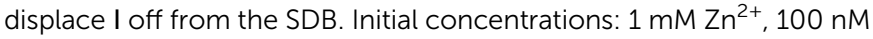
SDB, $1000 \mathrm{nM} \mathrm{S}, 400 \mathrm{nM} \mathrm{I}$, and $400 \mathrm{nM} \mathrm{I*}$. 
Changing the hybridization state of the catalytic core allows dynamic and allosteric control of the cleavage rate (Fig. 2B). First, an inactive $\operatorname{SDB}(n=12)$ is added in $\mathbf{S}$ solution (step 1), and the cleavage rate is very slow since the catalytic core is almost completely sequestered by the blocking sequence. By adding complementary initiator (I), the blocking sequence is displaced from the catalytic core (step 2), which resulted in dramatically increasing of the cleavage rate. Also, when the complementary strand $\mathbf{I}^{*}$ is added, the cleavage reaction can be effectively stopped by removing I from the blocking sequence through forming I-I* duplex. Therefore, the above results confirm that the cleavage kinetics of proposed SDB can be tuned by modulating the blocking strength and hybridization state of the catalytic core of $17 \mathrm{E}$.

\section{Fine adjustment of the cleavage rate}

Fine adjustment of the cleavage rate can be made by changing the blocking site. Serials of SDBs with catalytic cores sequestered at $3^{\prime}, 5^{\prime}$, and center were designed respectively. As shown in Fig. 3A, lengthening $n$ from 0 to $14 \mathrm{nt}$ slows the cleavage rates

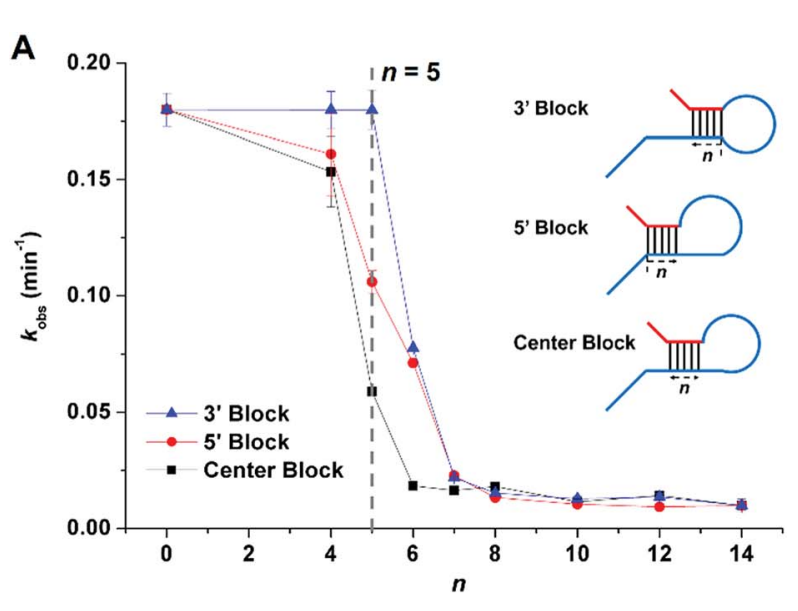

B

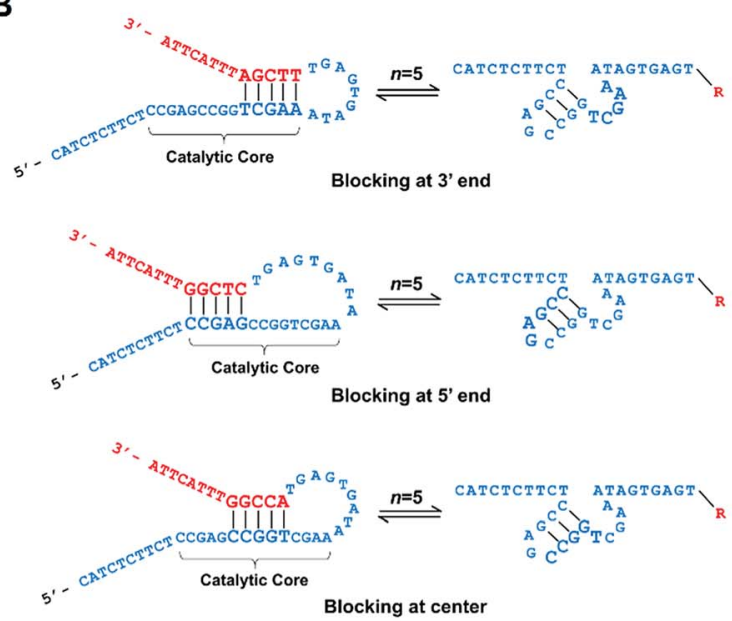

Fig. 3 (A) The effect of binding lengths and blocking sites on cleavage kinetics. Inset shows proposed secondary structures of SDBs. The error bars represent the standard deviation of three measurements. (B) The proposed second structures of SDBs with 5 bases blocking at $3^{\prime}$ end, $5^{\prime}$ end, and center. from 0.19 to $0.0093 \mathrm{~min}^{-1}$. Notably, when the same number of bases of the catalytic core are hybridized, different cleavage rates are observed for SDBs with catalytic cores sequestered at $3^{\prime}, 5^{\prime}$, and center. Take 5 bases blocking as example, the cleavage rate of SDB sequestered at $3^{\prime}$ end of the catalytic core is the fastest $\left(k_{\mathrm{obs}}=0.18 \mathrm{~min}^{-1}\right)$, and that is slowest at center $\left(k_{\mathrm{obs}}=\right.$ $\left.0.059 \mathrm{~min}^{-1}\right)$. Earlier studies pointed out that the unpaired $\mathrm{T}$ and three $\mathrm{G} / \mathrm{C}$ pairs in the catalytic core play crucial roles in 17E. ${ }^{38}$ First, the $\mathrm{T}$ acts as spacer between the neighboring $\mathrm{C}$ and the short $\mathrm{G} / \mathrm{C}$ paired stem to ensure that $\mathrm{C}$ is at the most adequate position to carry out the catalytic function. ${ }^{39}$ In addition, the three G/C pairs promote activity due to higher stem stability. ${ }^{40}$ Hence, comparing with SDBs sequestered at $3^{\prime}$ and $5^{\prime}$, center blocking (CCGGT) inhibits both the unpaired $\mathrm{T}$ and the three-G/C paired stem (Fig. 3B), which results in the slowest cleavage rate. We demonstrate that the proposed SDBs enable flexible control of the cleavage kinetics.

To further demonstrate the power of our design strategy, we applied the sequestered DNAzyme beacon strategy to couple with a divalent metal-dependent DNAzyme, 10-23 DNAzyme. ${ }^{\mathbf{4 1 , 4 2}}$ Serials of SDBs with catalytic cores sequestered at center were designed respectively. As shown in Fig. 4, in a fixed concentration of $\mathrm{Pb}^{2+}(100 \mu \mathrm{M})$, lengthening $n$ from 1 to $15 \mathrm{nt}$ slows the cleavage rates from 0.217 to $0.0006 \mathrm{~min}^{-1}$. These results demonstrate that the sequestered DNAzyme beacon strategy presented can be extended to couple with other RNA-cleaving DNAzymes to enable flexible control of the cleavage kinetics without changing the concentrations of mental ions.

\section{Control of cleavage rate by tuning the addition of triggering stimulator}

The cleavage kinetics of SDBs can also be finely regulated by tuning the concentration of additional triggering stimulator, which can be applied to target DNA detection. As shown in Fig. 5A, a completely sequestered DNAzyme beacon was

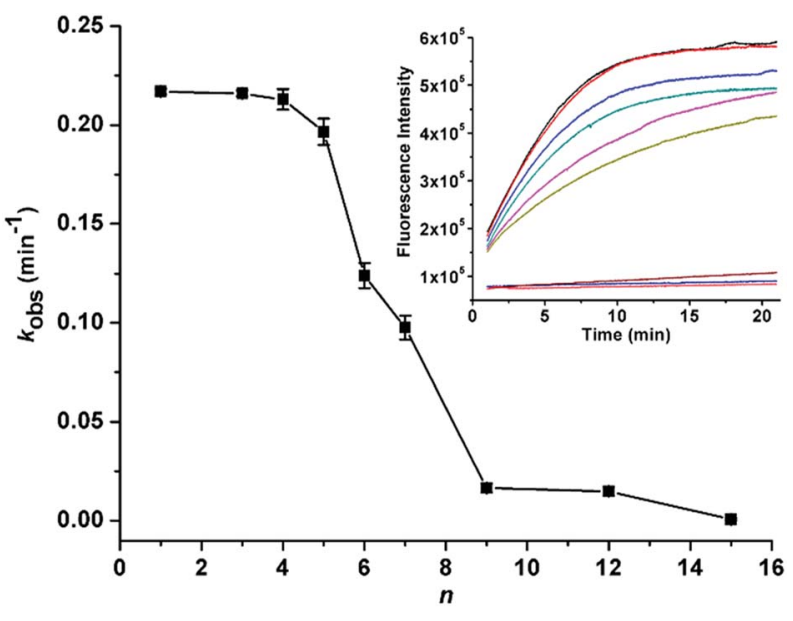

Fig. 4 The effect of binding lengths on 10-23 DNAzyme cleavage kinetics. The error bars represent the standard deviation of three measurements. Inset shows the change in fluorescence in the presence of different length blocking: 0, 1, 3, 4, 5, 6, 7, 9, 12, and $15 \mathrm{nt}$ from bottom to top, respectively. Initial concentrations: $100 \mu \mathrm{M} \mathrm{Pb}^{2+}$, $100 \mathrm{nM}$ SDB, and $500 \mathrm{nM} \mathrm{S}$. 

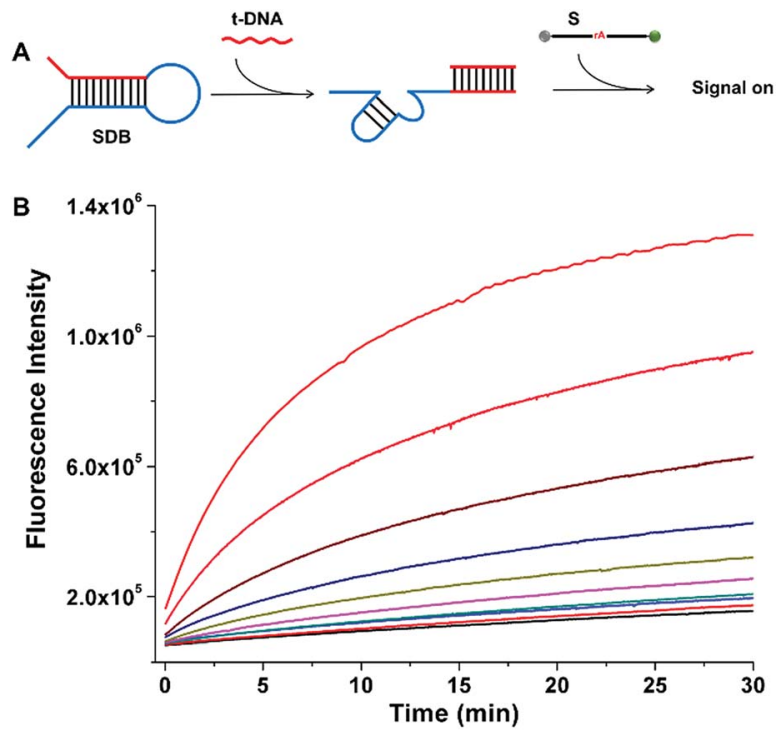

Fig. 5 Control of cleavage rates through control of stimulator concentration. (A) Schematic representation of the SDB activated by the addition of DNA trigger. (B) The effect of t-DNA concentrations on the catalytic reaction rates. The curves show the change in fluorescence in the presence of different concentrations of t-DNA (nM): 0 , $0.5,1,2,5,10,15,20,50$, and 100 from bottom to top, respectively.

designed. The DNA trigger (t-DNA) with specific sequence is complementary to the sequence of the red domain with an overhang toehold in SDB. In the absence of t-DNA, the 17E DNAzyme (blue domain) is sequestered by the hybridization of catalytic core, which provides a low background fluorescence. In the presence of t-DNA, the 17E DNAzyme is liberated from the caged structure with catalytic activity being restored. The activate $17 \mathrm{E}$ DNAzyme can catalyze the cleavage of the substrate, which resulting in an obvious increase of fluorescent signal. As the concentration of t-DNA increases from 0.5 to $100 \mathrm{nM}$, the fluorescence intensity generated by the cleavage of substrate also increases (Fig. 5B), which indicates that the cleavage reaction can be enhanced in a t-DNA-concentration-dependent way (Fig. S1†). Therefore, it is demonstrated that the presence of t-DNA can enhance the catalytic activity of the DNAzyme, and the kinetics of the cleaving reaction can be regulated by tuning the addition of triggering stimulator.

\section{SDB-based dual stem-loop structure probe for arbitrary target sequence biosensing}

To further demonstrate the universality of SDB in the applications of nucleic acid analysis. We designed a general sensing strategy for the detection of arbitrary target sequence (Fig. 6A). A general probe (DS-probe) was designed using a metastable dual stem-loop structure. DS-probe comprises several domains that make up recognition and signalling modules (i.e. SDB). The inner 6 bp stem (red domain) weakly sequesters the 17E DNAzyme by forming a SDB through hybridizing with the catalytic core. The outer stem (green domain) stabilizes the structure and is available for arbitrary target recognition. In the absence of target DNA (target), the weakly sequestered SDB is stabilized by the synergistic dual stem-loop structure, and is inactive to
A

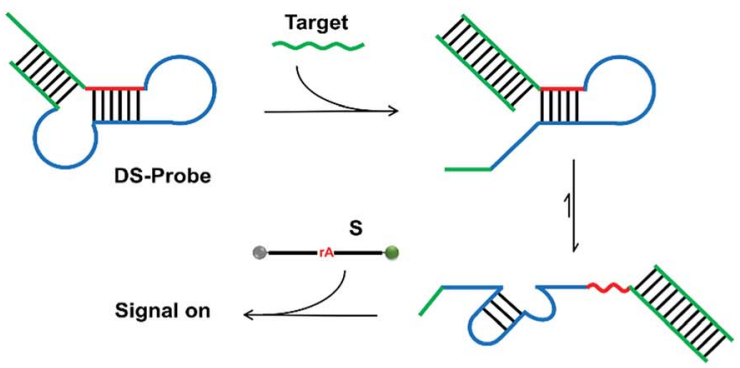

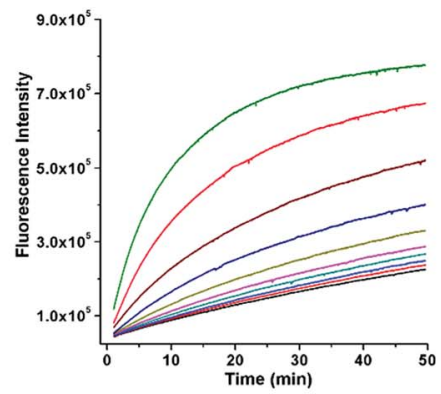

C

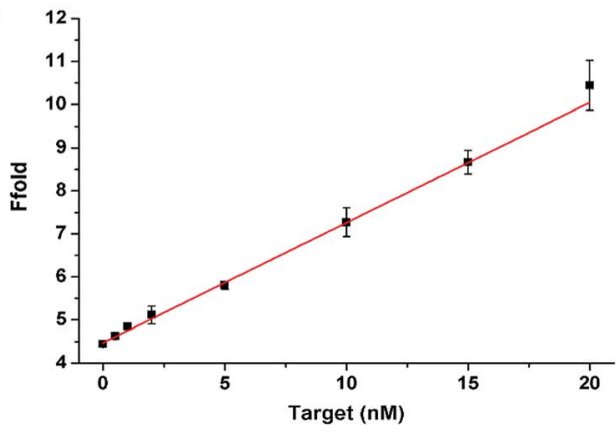

D

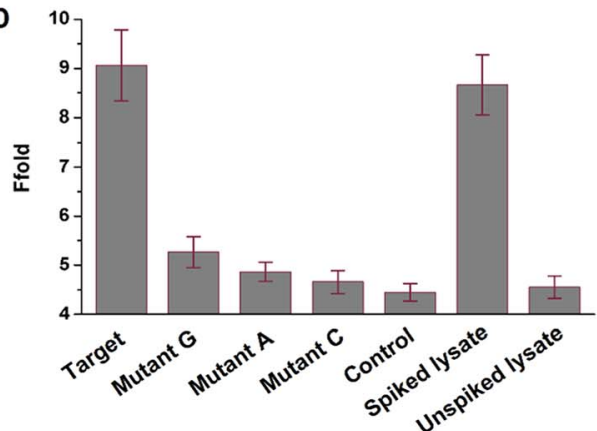

Fig. 6 Sensing system for the detection of arbitrary target sequence. (A) Schematic representation of the general target sensing strategy. A metastable dual stem-loop structure probe was designed by combining recognition and DNAzyme modules. (B) Time-dependent fluorescence response on varying concentrations of target. The curves show the change in fluorescence in the presence of different concentrations of the target (nM): $0,0.5,1,2,5,10,15,20,50$, and 100 from bottom to top, respectively. (C) The linear responses of fluorescence enhancement at low target concentrations. (D) Fluorescence enhancement of the sensing system for a series of targets ( $15 \mathrm{nM}$ ): target sequence, single-base mutant sequences, control sample, and target sequence spiked or unspiked in 5-fold diluted HeLa cell lysates, respectively. The error bars represent the standard deviation of three measurements. 
substrate reporter. In the presence of target, the outer stem is opened through toehold-mediated strand-displacement reaction with target. The relatively weak SDB freed by target recognition allows it to interconvert between hairpin and catalyst forms, which resulting in an obvious signal increase arised from substrate cleavage. Fig. 6B shows the time-dependent fluorescence changes upon analyzing different concentrations of target. As the concentration of the analyte increases from 0.5 to $100 \mathrm{nM}$, the fluorescence intensity generated by the cleavage of substrate also increases. A linear correlation is obtained in the range of 0-20 $\mathrm{nM}$ with a detection limit of $0.13 \mathrm{nM}$ based on $3 \sigma /$ slope ( $\sigma$, standard deviation of the blank samples) (Fig. 6C).

To quantitatively evaluate the specificity of the proposed sensing system, a series of single-base mismatch sequences of target were tested to obtain the corresponding discrimination factors. The discrimination factor is defined as the ratio of the signal gain obtained with the complementary target DNA to that obtained with the mismatch sequence under the same conditions. As shown in Fig. 6D, the discrimination factors are 5, 9, and 17 for $\mathrm{T}>\mathrm{G}, \mathrm{T}>\mathrm{A}$, and $\mathrm{T}>\mathrm{C}$ substitutions, respectively, clearly demonstrating the good selectivity of the sensing system. To evaluate the robustness of the sensing system in the physiological medium, a 5-fold diluted HeLa cell lysate was spiked with the target DNA. The spiked recovery of $15 \mathrm{nM}$ target DNA is $95 \%$ with a RSD of $7 \%$ ( $n=3$; Fig. 6D). Meanwhile, no evident fluorescence enhancement is observed using an unspiked lysate sample, indicating the possibility for biological sample applications. Therefore, the above results together demonstrate the universality of SDB in the applications of nucleic acid biosensing. By utilizing these strategies, DNAzymes with desired catalytic activity can be applied to biosensors and DNAzyme-based nanodevices.

\section{Conclusions}

In conclusion, we have developed a sequestered DNAzyme beacon strategy that allows flexible control of DNAzyme catalytic activities in DNA structures. The crucial element of the proposed strategy is that the catalytic cores of DNAzymes are precisely blocked by designed hybridization. As a result, the cleavage kinetics of proposed SDB can be finely tuned by over one order of magnitude via modulating the blocking strength and the concentration of triggering stimulator. The sequestered DNAzyme beacon strategy was further successfully employed to construct a dual stem-loop structure probe for the detection of arbitrary target sequence. DNAzyme is an important basis of many DNA nanodevices. We expect the additional design flexibility provided by the sequestered DNAzyme beacons to contribute to the development of DNAzymebased nanodevices, such as biosensors.

\section{Conflicts of interest}

There are no conflicts to declare.

\section{Acknowledgements}

This work was financially supported by the National Natural Science Foundation of China (21605134).

\section{Notes and references}

1 S. K. Silverman, Trends Biochem. Sci., 2016, 41, 595.

2 R. R. Breaker, Nat. Biotechnol., 1997, 15, 427.

3 Y. Lu, Chem.-Eur. J., 2002, 8, 4588.

4 G. F. Joyce, Annu. Rev. Biochem., 2004, 73, 791.

5 J. C. Achenbach, W. Chiuman, R. P. G. Cruz and Y. Li, Curr. Pharm. Biotechnol., 2004, 5, 312.

$6 \mathrm{~J}$. Liu and Y. Lu, Angew. Chem., Int. Ed., 2007, 46, 7587.

7 L. M. Lu, X. B. Zhang, R. M. Kong, B. Yang and W. Tan, J. Am. Chem. Soc., 2011, 133, 11686.

8 X. H. Zhao, L. Gong, X. B. Zhang, B. Yang, T. Fu, R. Hu, W. Tan and R. Yu, Anal. Chem., 2013, 85, 3614.

9 L. Freage, A. Trifonov, R. Tel-Vered, E. Golub, F. Wang, J. S. McCaskill and I. Willner, Chem. Sci., 2015, 6, 3544.

10 L. Gong, Z. Zhao, Y. F. Lv, S. Y. Huang, T. Fu, X. B. Zhang,

G. L. Shen and R. Q. Yu, Chem. Commun., 2015, 51, 979.

11 W. Zhou, R. Saran and J. Liu, Chem. Rev., 2017, 117, 8272.

12 A. V. Garibotti, S. M. Knudsen, A. D. Ellington and N. C. Seeman, Nano Lett., 2006, 6, 1505.

13 X. Mao, A. J. Simon, H. Pei, J. Shi, J. Li, Q. Huang, K. W. Plaxco and C. Fan, Chem. Sci., 2016, 7, 1200.

14 M. N. Stojanovic, S. Semova, D. Kolpashchikov, J. Macdonald, C. Morgan and D. Stefanovic, J. Am. Chem. Soc., 2005, 127, 6914.

15 J. Elbaz, O. Lioubashevski, F. Wang, F. Remacle, R. D. Levine and I. Willner, Nat. Nanotechnol., 2010, 5, 417.

16 J. Elbaz, F. Wang, F. Remacle and I. Willner, Nano Lett., 2012, 12, 6049.

17 R. Orbach, B. Willner and I. Willner, Chem. Commun., 2015, 51, 4144.

18 R. Orbach, F. Remacle, R. D. Levine and I. Willner, Chem. Sci., 2014, 5, 1074.

19 Y. Tian, Y. He, Y. Chen, P. Yin and C. Mao, Angew. Chem., Int. Ed., 2005, 44, 4355.

20 K. Lund, A. J. Manzo, N. Dabby, N. Michelotti, A. JohnsonBuck, J. Nangreave, S. Taylor, R. Pei, M. N. Stojanovic, N. G. Walter, E. Winfree and H. Yan, Nature, 2010, 465, 206.

21 R. Pei, S. K. Taylor, D. Stefanovic, S. Rudchenko, T. E. Mitchell and M. N. Stojanovic, J. Am. Chem. Soc., 2006, 128, 12693.

22 Y. Tang, B. Ge, D. Sen and H. Z. Yu, Chem. Soc. Rev., 2014, 43, 518.

23 H. Lin, Y. Zou, Y. Huang, J. Chen, W. Y. Zhang, Z. Zhuang, G. Jenkins and C. J. Yang, Chem. Commun., 2011, 47, 9312.

24 Y. Huang, L. Fang, Z. Zhu, Y. Ma, L. Zhou, X. Chen, D. Xu and C. Yang, Biosens. Bioelectron., 2016, 85, 496.

25 Z. Zhang, D. Balogh, F. Wang and I. Willner, J. Am. Chem. Soc., 2013, 135, 1934.

26 G. Zhu, J. Zhang, E. Song, M. Donovan, K. Zhang, C. Liu and W. Tan, Proc. Natl. Acad. Sci. U. S. A., 2013, 110, 7998.

27 H. M. Meng, X. Zhang, Y. Lv, Z. Zhao, N. N. Wang, T. Fu, H. Fan, H. Liang, L. Qiu and G. Zhu, ACS Nano, 2014, 8, 6171.

28 W. H. Chen, X. Yu, A. Cecconello, Y. S. Sohn, R. Nechushtai and I. Willner, Chem. Sci., 2017, 8, 5769.

29 H. Fan, X. Zhang and Y. Lu, Sci. China: Chem., 2017, 60, 591. 
30 W. Zhou, J. Ding and J. Liu, Theranostics, 2017, 7, 1010.

31 R. R. Breaker and G. F. Joyce, Chem. Biol., 1994, 1, 223.

32 J. Li and Y. Lu, J. Am. Chem. Soc., 2000, 122, 10466.

33 J. Liu, A. K. Brown, X. Meng, D. M. Cropek, J. D. Istok, D. B. Watson and Y. Lu, Proc. Natl. Acad. Sci. U. S. A., 2007, 104, 2056.

34 S. W. Santoro, G. F. Joyce, K. Sakthivel, S. Gramatikova and C. F. Barbas, J. Am. Chem. Soc., 2000, 122, 2433.

$35 \mathrm{~J}$. Li, W. Zheng, A. H. Kwon and Y. Lu, Nucleic Acids Res., 2000, 28, 481.

36 S.-F. Torabi, P. Wu, C. E. McGhee, L. Chen, K. Hwang, N. Zheng, J. Cheng and Y. Lu, Proc. Natl. Acad. Sci. U. S. A., 2015, 112, 5903.
37 W. Zhou, M. Vazin, T. Yu, J. Ding and J. Liu, Chem.-Eur. J., 2016, 22, 9835.

38 W. Zhou, Y. Zhang, J. Ding and J. Liu, ACS Sens., 2016, 1, 600.

39 B. Wang, L. Cao, W. Chiuman, Y. Li and Z. Xi, Biochemistry, 2010, 49, 7553.

40 A. Peracchi, M. Bonaccio and M. Clerici, J. Mol. Biol., 2005, 352, 783.

41 S. W. Santoro and G. F. Joyce, Biochemistry, 1998, 37, 13330.

42 Q. Wang, D. Zhang, M. Cheng, J. He and K. Liu, Acta Pharm. Sin. B, 2012, 2, 28. 